\title{
EXTRACCIÓN POR ACCIÓN BIOCATALÍTICA Y CUANTIFICACIÓN DE LUTEÍNA DE TOMATE DE ÁRBOL (Cyphomandra betacea)
}

\author{
Extraction by biocatalytic action and quantification of lutein from tomato of tree \\ (Cyphomandra betacea)
}

Javier S. Córdova ${ }^{2,3}$, Blanca L. Roque ${ }^{1}$

${ }^{1}$ Facultad de Ingeniería en Industrias Alimentarias, Universidad Nacional del Centro del Perú. ${ }^{2}$ Facultad de Farmacia y Bioquímica, Universidad Nacional Mayor de San Marcos. ${ }^{3}$ Centro Latinoamericano de Enseñanza e Investigación en Bacteriología Alimentaria - CLEIBA

\section{RESUMEN}

Se determinó la cantidad de oleorresina y se cuantificaron los componentes bioactivos, mediante un cromatógrafo líquido de alta eficiencia (HPLC), obteniéndose en los cromatogramas, luteína, betacaroteno y licopeno en gran cantidad. Se acondicionaron los frutos, previa determinación del índice de madurez -que fue de 4,71-, seguidamente se deshidrataron a $40^{\circ} \mathrm{C}$ por 96 horas en un secador de bandejas y se sometieron a molienda y tamizado con malla de diámetro menor a $425 \mu \mathrm{m}$. Posteriormente se hidrolizaron las paredes celulares del tejido seco para extraer luteína, utilizando un complejo enzimático de celulasa. Luego se realizó un proceso de lixiviación empleando una mezcla de hexano, etanol, acetona y tolueno en relación 10:6:7:7 (v/v/v/v) a temperatura de $35^{\circ} \mathrm{C}$ por 3 horas. El tratamiento que mejores resultados dio en la extracción de luteína, fue el tercero, que se realizó a $30^{\circ} \mathrm{C}$ por 4 horas con una relación sustrato:enzima de 1:16. Se obtuvo un rendimiento para luteína de 98,30 mg/10og materia seca. Los picos cromatográficos de luteína, $\beta$-caroteno y licopeno identificados, presentan espectros característicos con longitudes de onda de máxima absorción de 446, 452 y $471 \mathrm{jm}$, respectivamente.

Palabras clave: Extracción biocatalítica, luteína, carotenoides, tomate de árbol.

\section{SUMMARY}

The amount of oleoresin was determined and the bioactive components were quantified by high efficiency liquid chromatograph (HPLC) obtaining, in the chromatograms, lutein, beta-carotene and licopene in large quantities. After determination of fruit maturity index, the fruits were conditioning -it was 4,71-; then were dehydrated at $40^{\circ} \mathrm{C}$ for 96 hours on a tray dryer, and were ground and sieved by) a mesh with diameter smaller than $425 \mu \mathrm{m}$. Subsequently, the cell walls were hydrolyzed to extract lutein from dry tissue using a cellulose enzyme complex. Following leaching was performed using a mixture of hexane, ethanol, acetone and toluene in a ratio of 10:6:7: $7(\mathrm{v} / \mathrm{v} / \mathrm{v} / \mathrm{v})$ at a temperature of $35^{\circ} \mathrm{C}$ for a time of 3 hours. The third treatment gave better results in the extraction of lutein, this was performed at $30^{\circ} \mathrm{C}$ for 4 hours and substrate:enzyme ratio 1:16. A yield of 98,30 mg lutein / $100 \mathrm{~g}$ of dry matter was obtained. The identified chromatographic peaks of lutein, $\beta$-carotene and lycopene, had characteristic spectra with wavelengths of maximum absorption of 446, 452 and $471 \mathrm{jm}$, respectively.

Keywords: Biocatalytic extraction, lutein, carotenoids, tree tomato.

\section{INTRODUCCIÓN}

L 1 tomate de árbol o tamarillo (Cyphomandra betacea S.) es originario de los Andes del Perú, por lo general crece en forma nativa en huertos. Asimismo se produce desde el norte de Argentina hasta el sureste de México y en las Antillas desde 1000 hasta 3500 metros de altitud. El tomate de árbol posee una riqueza impresionante de nutrientes y compuestos bioactivos, que lo hacen un fruto de atractivo comercial, especialmente en Europa, teniendo muy poca consideración en el mercado nacional, debido al desconocimiento de su composición fitoquímica_que incluye toda una gama de carotenoides ( $\beta$-caroteno, licopeno, luteína, etc.) que poseen propiedades nutracéuticas de importancia en la nutrición y la salud ${ }^{(1)}$.

Dichos carotenoides están relacionados con el aumento del sistema inmune, disminución del riesgo de padecer enfermedades degenerativas ${ }^{(1,2)}$, prevención de la degeneración macular de la retina, disminución del riesgo de formación de cataratas, formación y proliferación de epitelios ${ }^{(1)}$.

La extracción extraer estos compuestos exige un proceso riguroso, debido a que son muy susceptibles a factores ambientales (temperatura, luz, oxígeno, etc.). La hidrólisis enzimática es un 


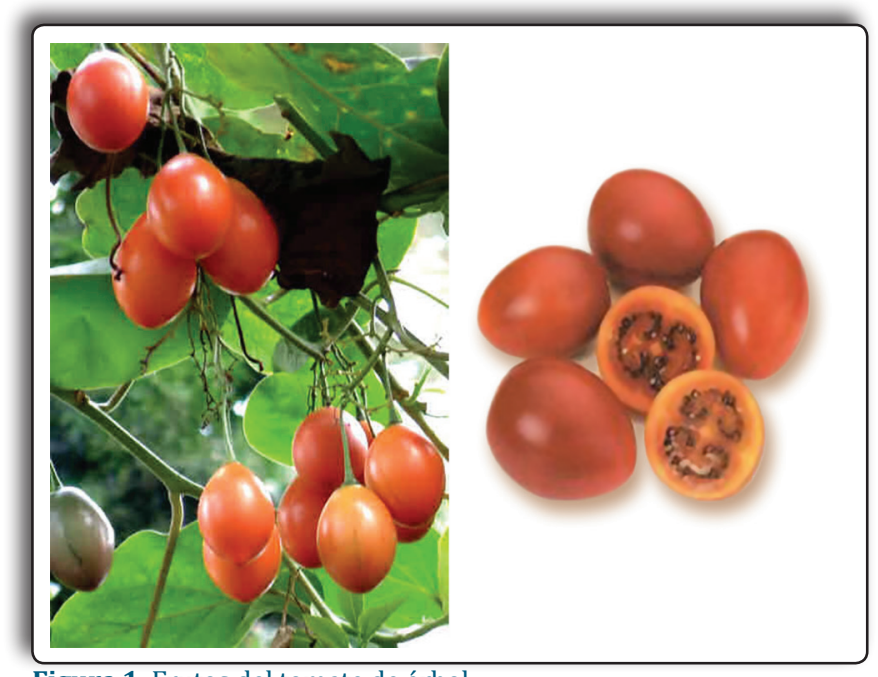

Figura 1. Frutos del tomate de árbol.

procedimiento adecuado, ya que degrada los tejidos celulósicos sin dejar residuos tóxicos y facilitando la salida de los compuestos bioactivos. Este proceso se da a temperaturas y tiempos moderados. Luego se debe realizar la solubilización de estos compuestos por lixiviación con organosolventes, también a condiciones moderadas.

El presente trabajo de investigación tuvo como objetivos: extraer y cuantificar, por acción biocatalítica, la luteína del tomate de árbol, y realizar la extracción y cuantificación de $\beta$-caroteno y licopeno por hidrólisis enzimática y organosolventes, comparando este método frente a una extracción convencional (soxhlet) y haciendo las cuantificaciones por Cromatografía Liquida de Alta eficiencia (HPLC).

\section{MATERIAL Y MÉTODOS}

\section{Material biológico}

Tomate deárbol, variedad anaranjada, procedente del distrito de Pariahuanca, provincia Huancayo, región Junín.

Complejo celulolítico sintetizado del hongo Aspergillus niger (ATCC 10864).

\section{Análisis fisicoquímico del tomate de árbol} fresco

Se realizaron los análisis de $\mathrm{pH}, \%$ acidez titulable (método potenciométrico) y sólidos solubles, para determinar el índice de madurez, siguiendo el método de Asociación Oficial de Químicos Analíticos (A.O.A.C.) ${ }^{(3)}$.

\section{Acondicionamiento de la materia prima}

Se utilizó el método de Hernández y Moreno (4), para el secado y acondicionamiento del tomate de árbol, el cual se llevó a cabo en un secador de bandejas por 96 horas a $45^{\circ} \mathrm{C}$, hasta una humedad de $13 \%$, posteriormente se molió el fruto, hasta llegar a un tamaño de partícula de $425 \mu \mathrm{m}$.

\section{Hidrólisis enzimática del tejido de tomate de árbol}

Se realizó la producción de enzimas celulóliticas de Aspergillus niger (ATCC 10864), según el método de Villena y Gutiérrez ${ }^{(5)}$. La hidrólisis enzimática se hizo de acuerdo al método de Salgado et al ${ }^{(6)}$. Se utilizaron tres gramos de muestra y, luego se trabajó bajo las siguientes condiciones: relación sustratoenzima (sto:enz) de 1:16 y 1:23; temperaturas de 30 y $40^{\circ} \mathrm{C}$, tiempos de 2 y 4 horas, y agitación constante de $190 \mathrm{rpm}$, luego se secó con aire caliente. El método descrito por Desikacharya et al (7), se utilizó para la solubilización del sustrato, con los organosolventes. Se hizo una mezcla de hexanoetanol-acetona-tolueno (HEAT) en relación al volumen (v/v/v/v) de $10 / 6 / 7 / 7$, respectivamente. La relación sustrato-solvente fue de 1:50, agitación de $190 \mathrm{rpm}$ y temperatura constante de $35^{\circ} \mathrm{C}$ por tres horas. Previa a la cuantificación se realizó el filtrado, la evaporación del solvente y finalmente la saponificación.

\section{Cuantificación de luteína}

Se realizo según el método descrito por Salgado et $a l^{(6)}$ y Candelas et $a l^{(8)}$. Se utilizó un Cromatógrafo Líquido de Alta Eficiencia (HPLC), con una columna C18, $5 \mu \mathrm{m}, 4,6$ x 250 mm, de Resterk. Se llevó una elución en condiciones isocráticas y en fase reversa,

Tabla 1. Resultados fisicoquímicos del tomate de árbol fresco.

\begin{tabular}{|c|c|c|c|c|}
\hline $\begin{array}{c}\% \text { Acidez } \\
\text { (ácido ć́trico) }\end{array}$ & $\begin{array}{l}\text { Sólidos solubles } \\
\text { totales ( }{ }^{\circ} \text { Brix) }\end{array}$ & pH & $\begin{array}{l}\text { Índice de } \\
\text { madurez }\end{array}$ & $\begin{array}{c}\text { Capacidad } \\
\text { antioxidante } \\
\text { mM eq Trolox } / g\end{array}$ \\
\hline $\begin{array}{c}2,55 \\
\pm \\
0,1\end{array}$ & $\begin{array}{c}12,0 \\
\pm \\
0,7\end{array}$ & $\begin{array}{l}3,88 \\
\pm \\
0,12\end{array}$ & $\begin{array}{c}4,87 \\
\pm \\
0,4 \\
\end{array}$ & $\begin{array}{c}803,21 \\
\pm \\
12,6\end{array}$ \\
\hline
\end{tabular}


con inyección de $0,5 \mathrm{~mL} /$ min; la mezcla del eluyente contenía el $60 \%$ de metanol, $38 \%$ de acetonitrilo y $2 \%$ de $2-$ propanol $(\mathrm{v} / \mathrm{v} / \mathrm{v})$. La separación se desarrolló a temperatura ambiente. Los pigmentos se monitorearon a 450 y $471 \mathrm{jm}$, empleando un detector de diodos de 1 s y $4 \mathrm{gm}$. Se inyectó a la columna $20 \mu \mathrm{L}$ de extracto de pigmento saponificado.

\section{RESULTADOS}

\section{Análisis fisicoquímico del tomate de árbol fresco}

En la tabla 1, se presenta el porcentajedeacidezquealcanzó el fruto seleccionado para los ensayos; esto conjuntamente con los resultados obtenidos de sólidos solubles y $\mathrm{pH}$ facilitaron determinar el grado de madurez.

\section{Hidrólisis celulolítica}

En la tabla 2, se presentan los resultados de la hidrólisis del tejido del tomate de árbol, en porcentajes de humedad. Los pesos de la torta seca hidrolizada comparados los pesos iniciales de la muestra, difieren en un promedio del $63 \%$ indicando que esta humedad es favorable para la interacción sustrato-solvente.

\section{Lixiviación con organosolvente}

La torta hidrolizada seca, fue sometida a lixiviación con solventes orgánicos. En la tabla 3, se observa el rendimiento (Rdto) de oleorresina (Óleo) obtenida en cada tratamiento; los factores de estudio fueron: relación ( $\mathrm{R}$ ) sustrato-enzima (sto:enz), temperatura ( $\mathrm{T})$ y tiempo $(\theta)$, los cuales, en sus
Tabla 2. Hidrólisis de tejido seco de tomate de árbol.

\begin{tabular}{cccc}
\hline TTo* hidrolítico & $\begin{array}{c}\text { Peso inicial de } \\
\text { muestra seca (g) }\end{array}$ & $\begin{array}{c}\text { Peso de la muestra } \\
\text { seca hidrolizada (g) }\end{array}$ & $\begin{array}{c}\text { Humedad de la } \\
\text { muestra seca } \\
\text { hidrolizada (\%) }\end{array}$ \\
\hline T1 & 3,0 & $1,039 \pm 0,065$ & $8,30 \pm 0,22$ \\
T2 & 3,0 & $1,038 \pm 0,064$ & $7,89 \pm 0,55$ \\
T3 & 3,0 & $1,086 \pm 0,012$ & $8,47 \pm 0,12$ \\
T4 & 3,0 & $1,089 \pm 0,045$ & $6,99 \pm 0,49$ \\
T5 & 3,0 & $1,098 \pm 0,020$ & $8,50 \pm 0,12$ \\
T6 & 3,0 & $1,036 \pm 0,040$ & $7,97 \pm 0,42$ \\
T7 & 3,0 & $1,056 \pm 0,025$ & $8,10 \pm 0,34$ \\
T8 & 3,0 & $1,095 \pm 0,025$ & $7,22 \pm 0,58$ \\
\hline
\end{tabular}

* Número de Tratamientos.
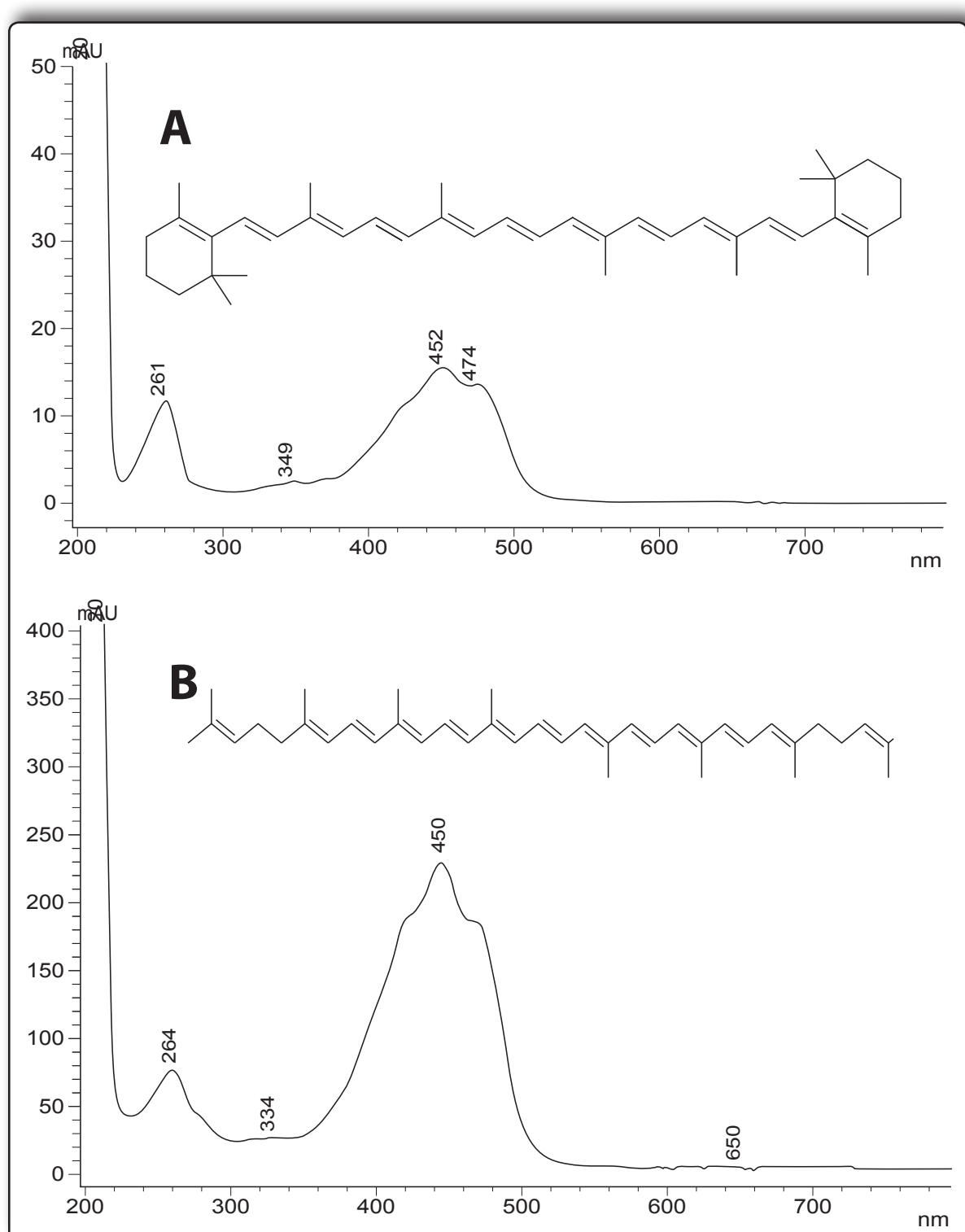

Figura 2. Perfiles de Luteina, $\beta$-caroteno y licopeno de oleorresina de tomate de árbol extraído por hidrólisis enzimática: (A) Patrón a 40 ppm y (B) Muestra tercer tratamiento (T3) $\left(30{ }^{\circ} \mathrm{C} / 4\right.$ $\mathrm{h} / 1: 16)$. 
diferentes niveles, muestran un marcado efecto en la extracción de oleorresina.

En la tabla 4, de los ocho tratamientos biocatalíticos seguidos por una lixiviación, a los que fue sometido el tejido de tomate de árbol, se observa que la cantidad de luteína obtenida en todos los tratamientos es superior a la obtenida cuando se extrae con el método convencional (soxhlet).

Es probable que durante la extracción se hayan conseguido muchos otros carotenoides, pero en menor cantidad.

En la figura 2, se observan los cromatogramas de los mejores tratamientos.

\section{DISCUSIÓN}

De la tabla 1 se observa que, la variación de la acidez disminuye conforme madura el fruto, siguiendo su evolución en relación inversa respecto al $\mathrm{pH}$ y los sólidos solubles. La leve disminución de la acidez a medida que maduran los frutos, se debe al consumo de los ácidos orgánicos en los procesos de síntesis de compuestos volátiles, los cuáles son los responsables del sabor del fruto ${ }^{(9)}$.

De la tabla 2, la hidrólisis se realizó en condiciones de oscuridad para evitar degradación y oxidación. Según Lehninger ${ }^{(9)}$, las condiciones no fueron agresivas, ya que la temperatura adecuada para una buena hidrólisis enzimática es menor a $45^{\circ} \mathrm{C}$. La extracción por este método y su solubilización con solvente orgánico, aplican temperaturas y tiempos moderados evitando el deterioro por oxidación o isomerización de los carotenoides.

Se puede indicar que la proporción de sustratoenzima (1:16) a $40^{\circ} \mathrm{C}$ y 2 horas de hidrólisis, empleados $\left.{ }^{\circ} \mathrm{C} / 4 \mathrm{~h} / 1: 16\right)$.
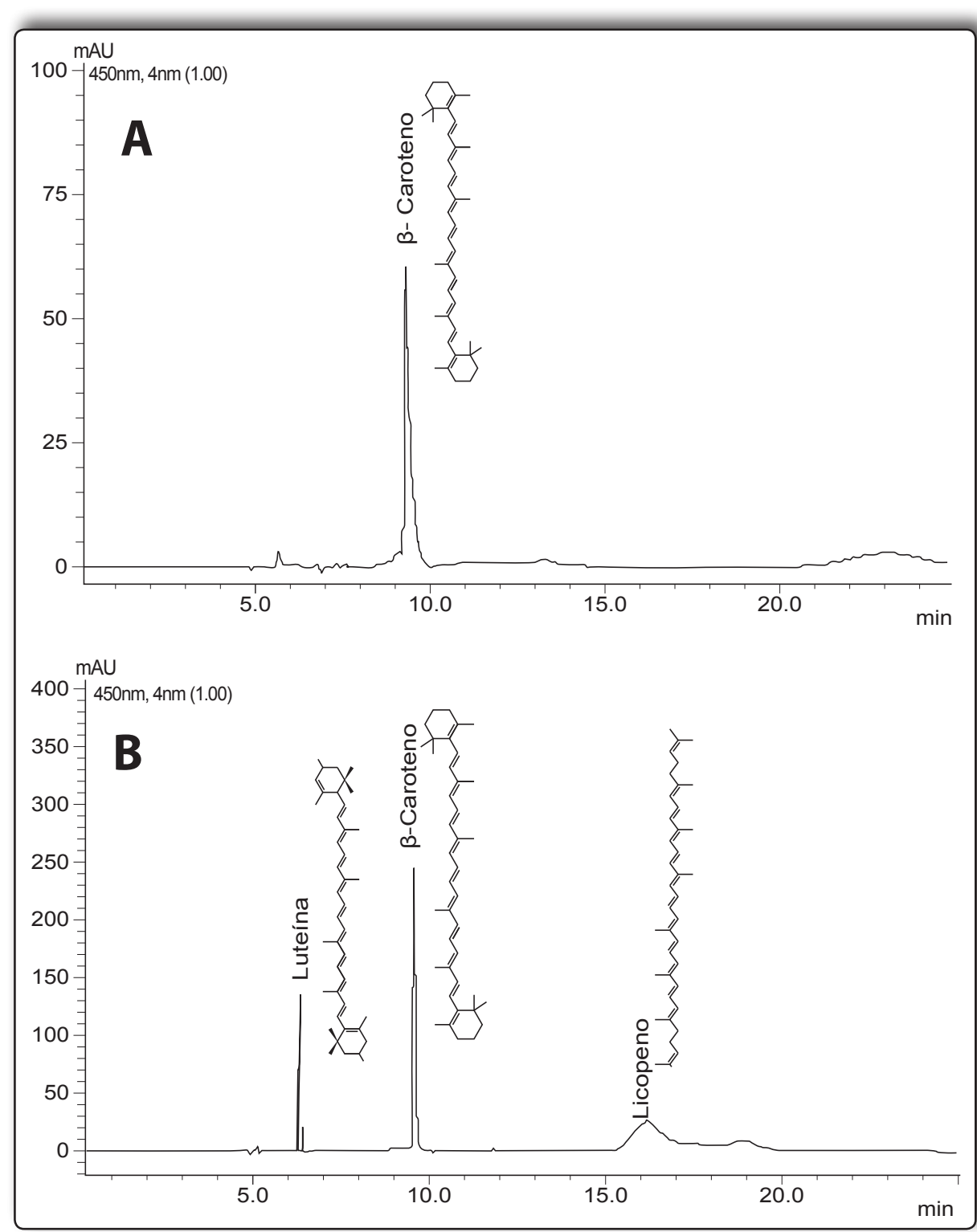

Figura 3. Cromatogramas de Luteína, $\beta$-caroteno y licopeno de oleorresina de tomate de árbol extraído por hidrólisis enzimática: (A) Patrón a 40 ppm y (B) Muestra tercer tratamiento (T3) (30

en el quinto tratamiento, permitieron degradar significativamente el tejido celular, con lo que se logró un mayor rendimiento, de aproximadamente 1,1 veces, comparado con el sexto tratamiento. Esto indica que el incremento de la relación de sustratoenzima a 1:23 no tiene mayor efecto la hidrólisis de la matriz vegetal, ya que una enzima funciona más eficiente cuando su concentración está en equilibrio con la del sustrato_con lo cual las colisiones "exitosas" con el complejo enzimático son más frecuentes, asegurando así que la mayor cantidad de enzima se encuentre activa. En estas condiciones, el producto 
Tabla 3. Rendimiento de oleorresina de tomate de árbol.

\begin{tabular}{cccccc}
\hline Tratamiento & $\begin{array}{c}\mathbf{R} \\
\text { (sto:enz) }\end{array}$ & $\begin{array}{c}\mathrm{T} \\
\left({ }^{\circ} \mathrm{C}\right)\end{array}$ & $\begin{array}{c}\boldsymbol{\theta} \\
(\mathbf{h})\end{array}$ & $\begin{array}{c}\text { Oleo } \\
(\mathbf{g} / \mathbf{g} \text { m,s })\end{array}$ & $\begin{array}{c}\text { Rdito } \\
(\%)\end{array}$ \\
\hline T1 & $1: 16$ & 30 & 2 & 0,467 & $15,57 \pm 0,6$ \\
T2 & $1: 23$ & 30 & 2 & 0,468 & $15,60 \pm 0,8$ \\
T3 & $1: 16$ & 30 & 4 & 0,489 & $16,30 \pm 1,0$ \\
T4 & $1: 23$ & 30 & 4 & 0,490 & $16,33 \pm 0,4$ \\
T5 & $1: 16$ & 40 & 2 & 0,495 & $16,48 \pm 0,1$ \\
T6 & $1: 23$ & 40 & 2 & 0,466 & $15,53 \pm 0,4$ \\
T7 & $1: 16$ & 40 & 4 & 0,476 & $15,87 \pm 0,2$ \\
T8 & $1: 23$ & 40 & 4 & 0,493 & $16,42 \pm 0,3$ \\
\hline
\end{tabular}

Tabla 4. Cuantificación de luteína de oleorresina de tomate de árbol.

\begin{tabular}{|c|c|c|c|c|c|c|c|}
\hline \multirow{2}{*}{ Tratamiento } & \multirow{2}{*}{$\underset{\text { (sto:enz) }}{\mathbf{R}}$} & \multirow{2}{*}{$\begin{array}{c}\theta \\
(\mathrm{h})\end{array}$} & \multirow{2}{*}{$\begin{array}{c}\mathrm{T} \\
\left({ }^{\circ} \mathrm{C}\right)\end{array}$} & \multirow{2}{*}{$\begin{array}{l}\text { Óleo } \\
\text { (g) }\end{array}$} & \multicolumn{3}{|c|}{ Luteína } \\
\hline & & & & & $(\mathrm{mg} / \mathrm{mL})$ & $100 \mathrm{~mL})$ & $100 \mathrm{~g} \mathrm{~m} / \mathrm{m} . \mathrm{s})$. \\
\hline $\mathrm{T} 1$ & $1: 16$ & 2 & 30 & 0,4670 & 0,050 & 18,38 & 61,27 \\
\hline $\mathrm{T} 2$ & $1: 23$ & 2 & 30 & 0,4680 & 0,052 & 18,47 & 61,57 \\
\hline T3 & $1: 16$ & 4 & 30 & 0,4890 & 0,120 & 37,14 & 98,30 \\
\hline $\mathrm{T} 4$ & $1: 23$ & 4 & 30 & 0,4900 & 0,089 & 27,06 & 90,20 \\
\hline $\mathrm{T} 5$ & $1: 16$ & 2 & 40 & 0,4945 & 0,099 & 31,64 & 95,47 \\
\hline T6 & $1: 23$ & 2 & 40 & 0,4660 & 0,060 & 17,78 & 59,27 \\
\hline $\mathrm{T} 7$ & $1: 16$ & 4 & 40 & 0,4760 & 0,070 & 22,60 & 75,37 \\
\hline T8 & $1: 23$ & 4 & 40 & 0,4925 & 0,090 & 29,42 & 90,06 \\
\hline Soxhlet & - & - & 60 & 0,7925 & 0,035 & 9,08 & 20,15 \\
\hline
\end{tabular}

(T6), con $15,53 \%$. No obstante, la extracción convencional (soxhlet) obtiene 1,6 veces más oleorrisina que la conseguida por hidrólisis enzimática y órganosolventes.

El tratamiento que mejores resultados dio en la extracción de luteína fue el tercero ( $\left.\mathrm{T}_{3}\right)$, llevado a cabo a $30^{\circ} \mathrm{C}$ por $4 \mathrm{~h}$ y con una relación sustrato:enzima de 1:16. En esas condiciones se obtuvieron $98,30 \mathrm{mg} / \mathrm{10og}$ materia seca, superando a la extracción convencional Soxhlet en 4,1 veces. En el tomate de árbol los picos cromatográficos de luteína, $\beta$-caroteno y licopeno identificados, presentan espectros característicos con longitudes de onda de máxima absorción de 446,

se obtiene a la máxima velocidad posible para la cantidad de enzima presente ${ }^{(10)}$.

La interacción temperatura y tiempo es significativa en el rendimiento de oleorresina, porque permite mejorar la distribución e incrementar el contacto sustrato-enzima aumentando el rendimiento y la cantidad de compuestos bioactivos debido a que generalmente un complejo enzimático posee efectos sinérgicos exo-endo y exo-exo ${ }^{(11)}$, lo que se traduce en una extracción óptima de los mencionados compuestos desde fuentes naturales.

De la figura $3 \mathrm{~B}$, el primer pico le correspondería a la luteína, identificada por su espectro y naturaleza ${ }^{(12)}$, el siguiente pico le correspondería al $\beta$-caroteno, que fue identificado en el mismo tiempo de retención que el patrón, y el tercer pico significativo correspondería al licopeno.

\section{CONCLUSIÓN}

El tratamiento que produjo mayor rendimiento de oleorresina, entre los ocho aplicados, fue el quinto ( $\left.\mathrm{T}_{5}\right)$, con $16,48 \%$, en comparación al menor rendimiento del sexto tratamiento
452 y $471 \mathrm{~nm}$, respectivamente.

\section{REFERENCIAS BIBLIOGRÁFICAS}

1. Van den Berg H, Faulks R, Granado HF, Hirschberg J, Olmedilla B, Sandmann G, et al. The potencial fot the improvement of carotenoid levels in food and the likely systemic effects. J Sci Food Agric 20oo; 8o(7): 880-912.

2. Fraser P, Bramley P. The biosynthesis and nutritional uses of carotenoids. Prog Lipid Res 2004; 43(3): 228-65.

3. AOAC. Official methods of analysis. $15^{\text {th }}$ ed. Association of Official Analytical Chemist. Washington, 1990.

4. Hernández G, Moreno M. Efecto del secado y del ácido cítrico sobre la degradación de los carotenoides de tamarillo (Cyphomandra betacea) Sendt). Ciencia y Tecnología Alimentaria 2000; 2(5): 228-33.

5. Villena G, Gutiérrez-Correa M. Production of cellulase by Aspergillus niger biofilms developed on polyester cloth. Letters in Applied Microbiology 2006; 43(3): 262-8.

6. Salgado M, Botello E, Rico R, Jiménez H, Cárdenas M, Navarrete J. Enzymatic treatment to improve extraction of capsaicinoids and carotenoids from chili (Capsicum annuum) fruits. J Agr Food Chem 2008; 56(21): $10012-8$. 
7. Desikacharya SSR, Naidu MM, Sowbhagya HB, Naik JP, Krishnamurthy N. inventors; Morgan \& Finnegan, L.L.P., assignee. Process of extracting chili (Capsicum) oleoresin. US. Patent Application 20,040,191,364. 2004.

8. Candelas MG, Alanís GM, Del Río F. Cuantificación de licopeno y otros carotenoides en tomate y polvo de tomate. Revista Mexicana de Agronegocios. 2006; 10(19): 1-12.

9. Lehninger A. Bioquímica. $2^{\text {da }}$ ed. Omega. Barcelona, 1999.

10. Badui S. Química de los alimentos. $4^{\text {ta }}$ ed. Pearson. México DF, 2006.

11. Ovando SL, Waliszewski KN. Preparativos de celulasas comerciales y aplicaciones en procesos extractivos. Universidad y Ciencia. 2005; 42(21): 113-22.

12. Khachik F, Englert G, Beecher GR, Smith JC. Isolation, structural elucidation, and partial synthesis of lutein dehydration products in extracts from human plasma. J Chromatogr B Biomed Appl 1995; 670(2): 219-33.

13. Cadoni E, Rita De Giorgi M, Medda E, Poma G. Supercritical $\mathrm{CO}_{2}$ extraction of lycopene and $\beta$-carotene from ripe tomatoes. Dyes and Pigments 1999; 44(1): 27-32.

Manuscrito recibido el: 16/07/14

Aceptado para su publicación el: 04/o9/2014

\section{Correspondencia}

Nombre: $\quad$ Ing. Javier Saúl Córdova Ramos

Dirección: Jr. Puno 1002 - Lima o1 - Perú

E-mail: Javier.cordova.ramos@hotmail.com 